\title{
The Role of Emotional Beliefs, Worry Behaviors, and Social Support in Cognitive, Physical and Behavioral Anxiety Syndrome
}

\section{Sohrab Amiri (PhD) ${ }^{1, *}$, Abolghasem Yaghobi (PhD) ${ }^{2}$, Tayebeh Bayat (BSc) ${ }^{3}$, Shamsi Shamshiri (BSc) ${ }^{3}$, Amir Ghasemi Navab (MSc) ${ }^{4}$}

${ }^{1}$ PhD of Psychology, Urmia University, Urmia, Iran.

${ }^{2}$ Associate Professor, Bu Ali Sina University, Hamedan, Iran

${ }^{3}$ MSc student in psychology, Bu Ali Sina University, Hamedan, Iran

${ }^{4}$ MSc in psychology, Alametabatbayi University, Tehran, Iran

* Corresponding Author: Sohrab Amiri, Urmia University, Urmia, Iran. Email: amirysohrab@yahoo.com

\section{Abstract}

Received: $15 / 06 / 2018$

Accepted: 08/10/2019

\section{How to Cite this Article:}

Amiri S, Yaghobi A, Bayat T, Shamshiri S, Ghasemi Navab A. The Role of Emotional Beliefs, Worry Behaviors, and Social Support in Cognitive, Physical and Behavioral Anxiety Syndrome. Pajouhan Scientific Journal. 2019; 17(2)

7-14. DOI: $10.29252 / p s j .17 .2 .7$
Background and Objective: Anxiety has a significant impact on academic, social performance and quality of life. This study examined the relationship between emotional beliefs, worry behaviors, and social support with cognitive, physical and behavioral aspects of anxiety.

Materials and Methods: For this purpose, from the statistical population of the Bu Ali Sina University students in 2018 year, 312 were selected by using multistage cluster sampling. Then, in order to assess objectives of the research, scale of measurement of emotional beliefs, worry behaviors, and social support and anxiety were distributed among the participants. Collected data were analyzed by using descriptive and correlation indicators and simultaneous regression.

Results: Results indicated that there is relationship between worry behaviors, emotional beliefs and social support with anxiety, so that there were a significant positive correlation between worry behaviors with cognitive, physical and behavioral aspects of anxiety $(\mathrm{P}<0.05)$. Also emotional beliefs with physical and behavioral aspects showed that a significant positive correlation $(\mathrm{P}<0.05)$. There were a significant negative correlation between social support with behavioral aspect of anxiety $(\mathrm{P}<0.01)$.

Conclusion: Individual differences in emotional aspects, worry and social support play an important role in the symptoms of Anxiety.

Keywords: Anxiety; Emotions; Social Support 
do: : $10.29252 /$ psj.17.2.7

\section{نقش باور هاى هيجانى، رفتار هاى نكرانى و حمايت اجتماعى در نشانكان شناختى، بدنى و رفتارى اضطراب}

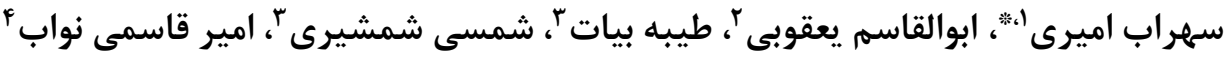
' ل دكترى تخصصى روانشناسى، دانشعاه اروميه، اروميه، ايران

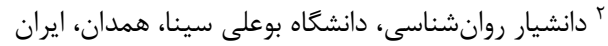

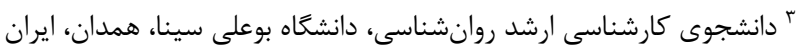

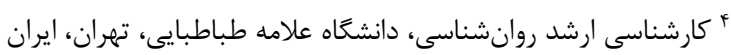
* نويسنده مسئول: سهراب اميرى، دانشخاه اروميه، اروميه، ايران. ايميل: amirysohrab@yahoo.com

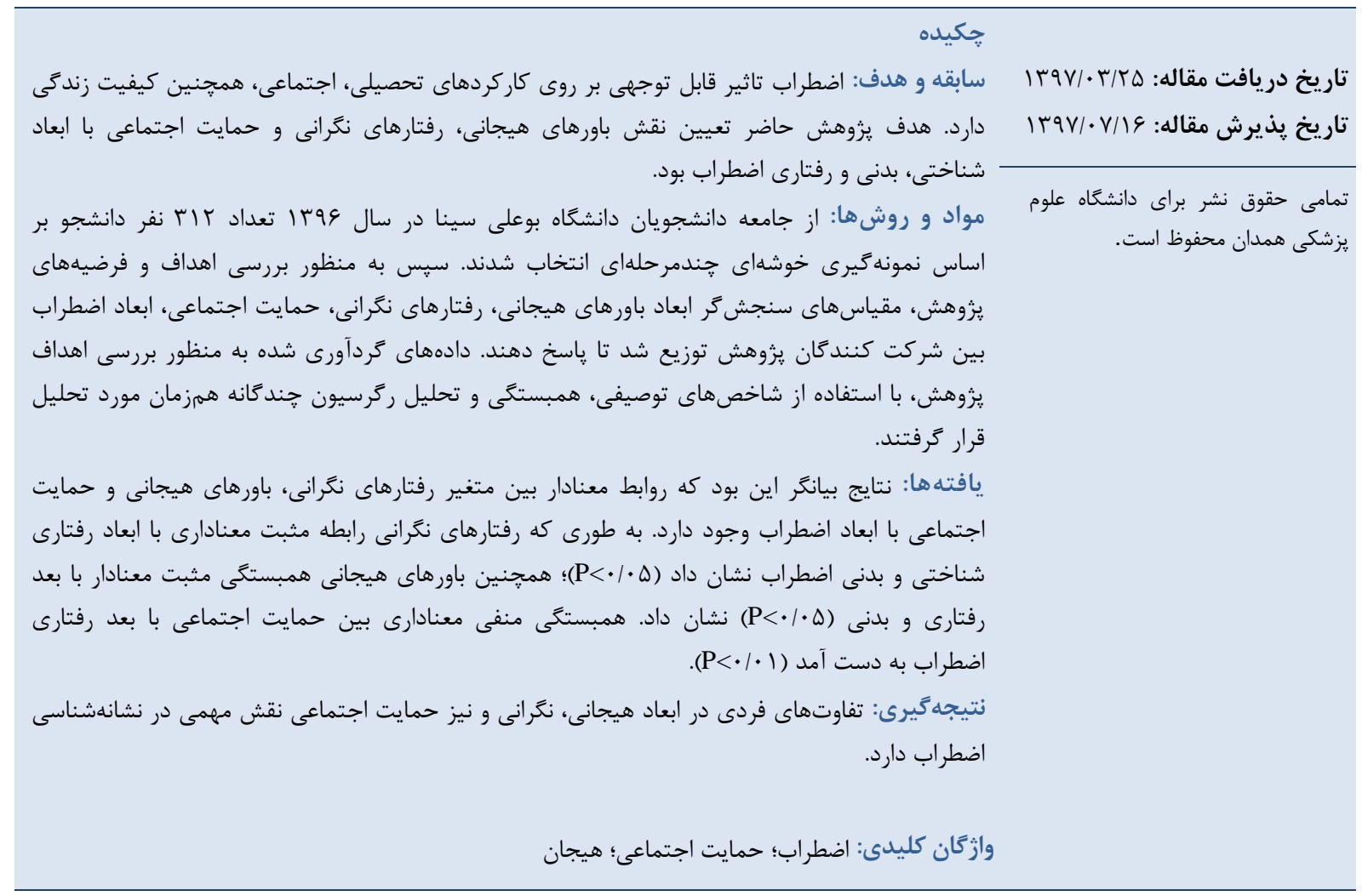

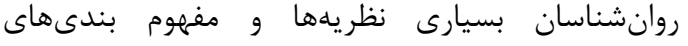

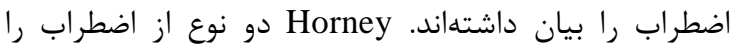

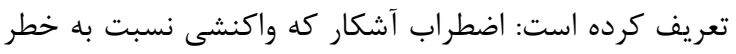

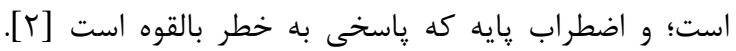

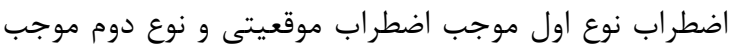

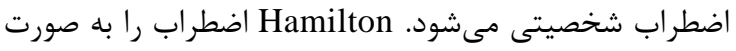
جسمانى، شامل شكايتهاى مرتبط با بدن، يا روانى شامل

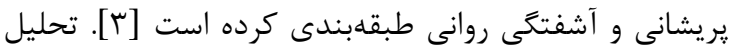

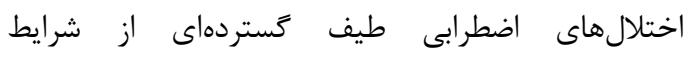

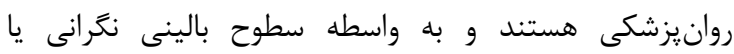

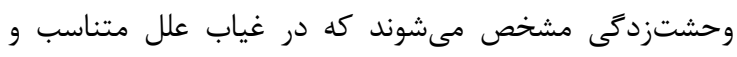

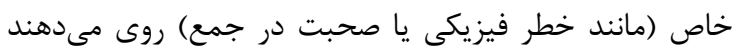

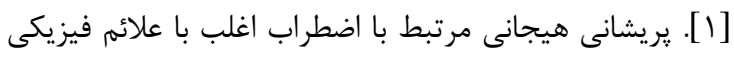

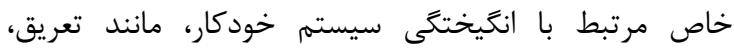
سرگيجه و تنفس سطحى، يا شكايتهاى جسمانى گستردهتر

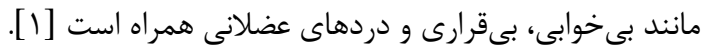


نظرى و بالينى است. رفتارهاى اجتنابى ناسازكارانه براى تعريف

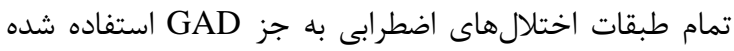

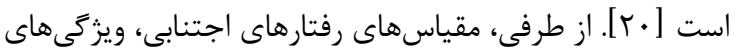

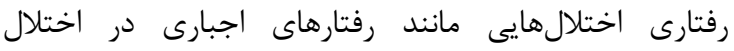

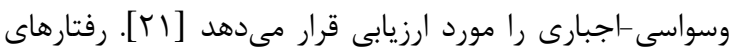

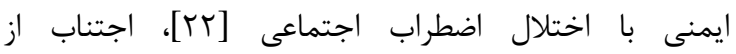

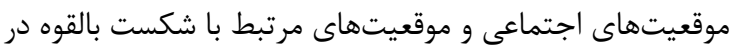

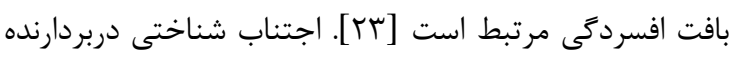

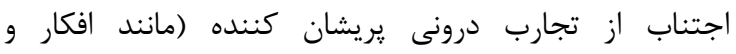

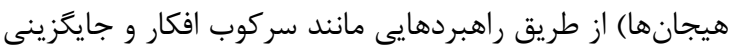

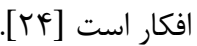
از طرفى، مطالعات اخير نشان داده است كه حمايت

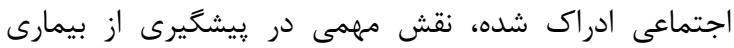

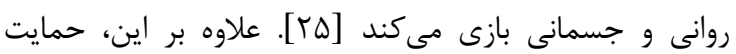

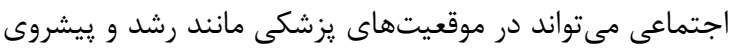

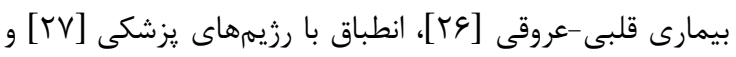

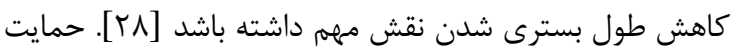

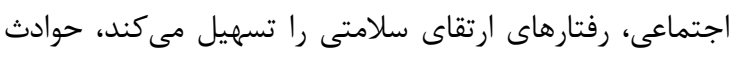

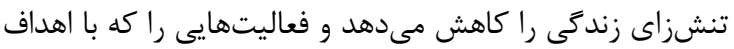

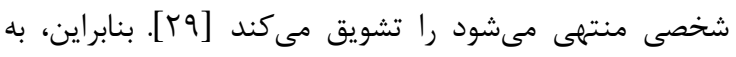

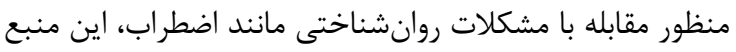

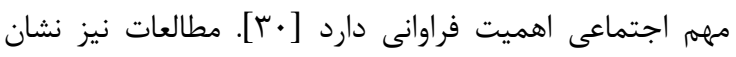

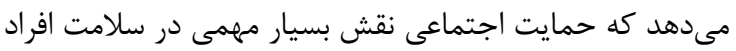

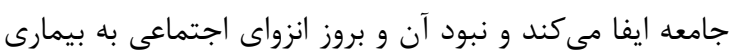



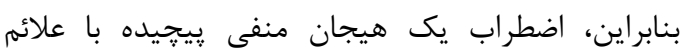

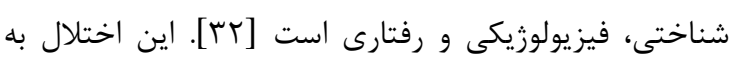

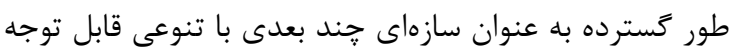

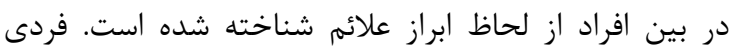

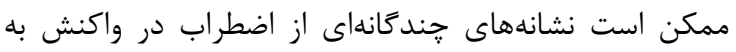

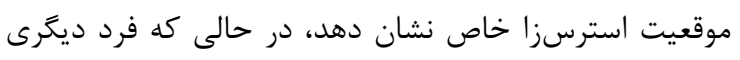

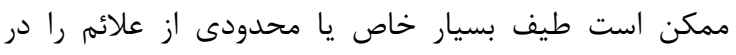

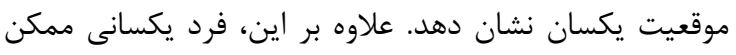

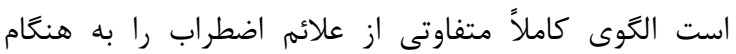

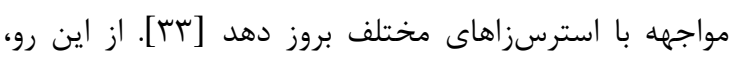

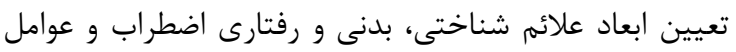

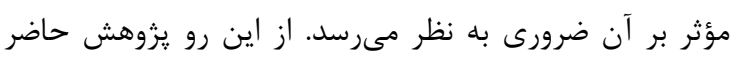

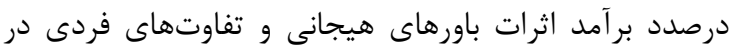

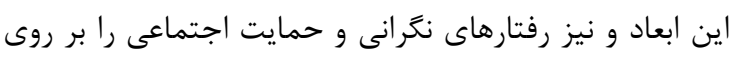
علائم شناختى، بدنى و رفتارى اضطراب مورد بر برسى و و مطالعه رئه

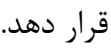

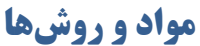
يزوهش حاضر با توجه به نوع متغيرها و شيوه كردآورى
عاملى انواع مقياسهاى اضطراب نشان مىدهد كه اضطراب

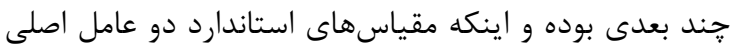

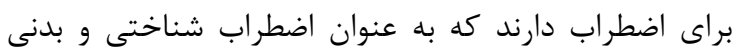

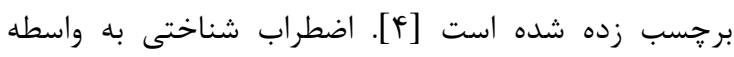

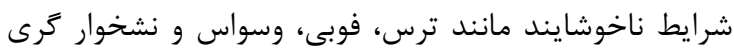

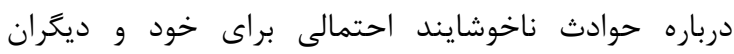

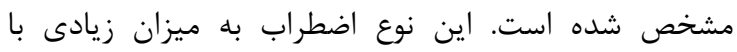

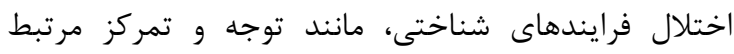

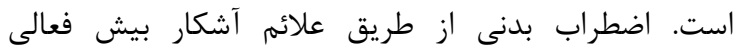

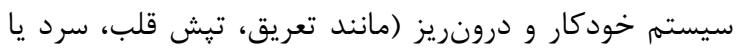

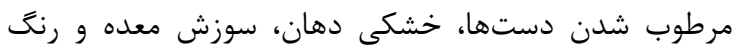

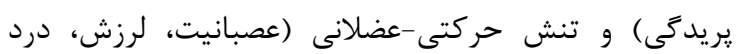

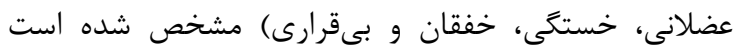

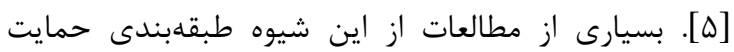

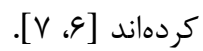
بنابر اين، اضطراب حالتى از انخيختىى هيجانى است كه

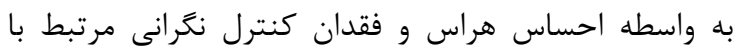

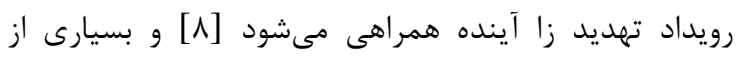

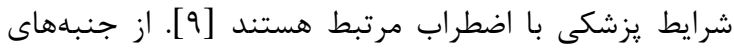

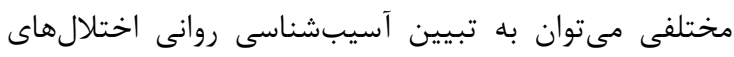

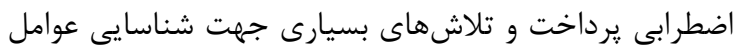

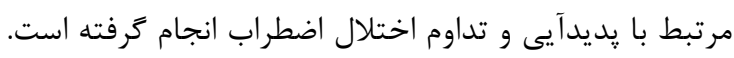

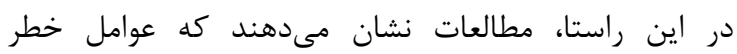

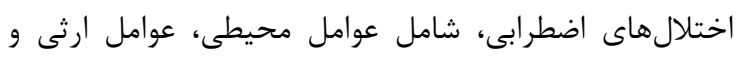

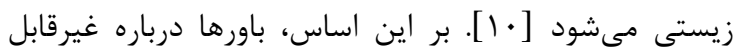

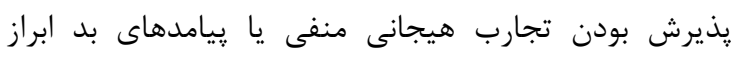

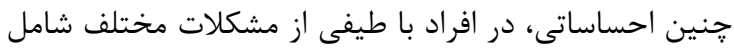

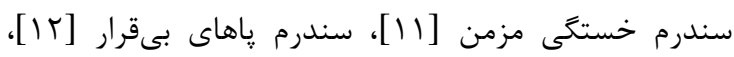

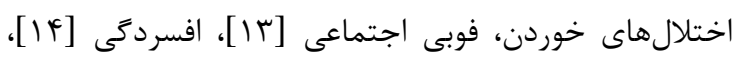

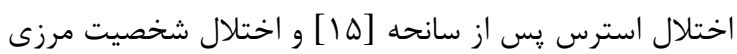

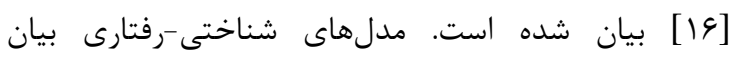

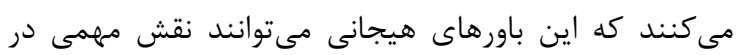

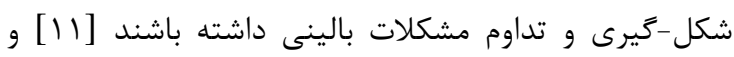

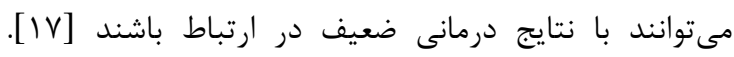

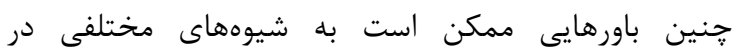

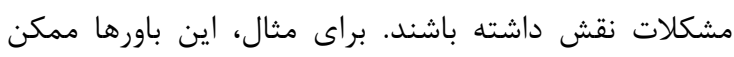


خودآكاهى و خود ادراكى را تضعيف نمايد [111]. اكر افكار

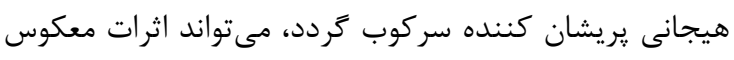

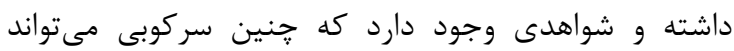

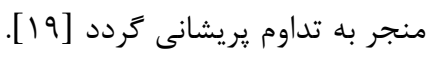

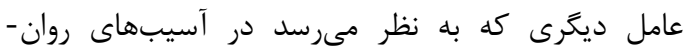
شناختى نقش دارد درى رفت رفتارهاى اجتنابى است كه بـ با اختلال اضطراب فراكير مرتبط هستند و داراى تلويحات تشخيصى، راتئ 
جيزها را توصيف مى كند يا خير، به سادكى اين موضوع را در

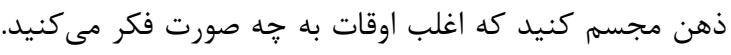

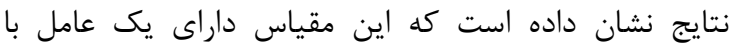

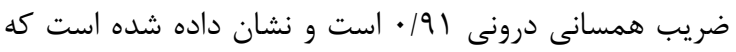

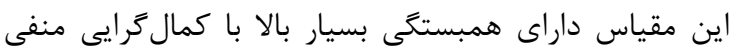

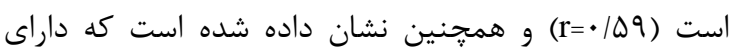

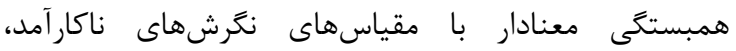

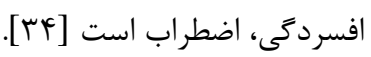

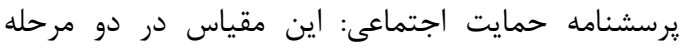

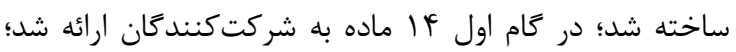

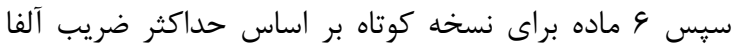
انتخاب كرديد. در مرحله دوم نسخه كورته كراه

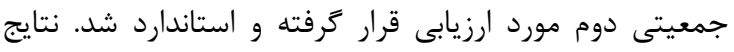

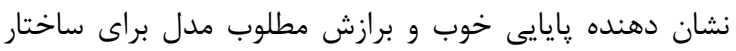



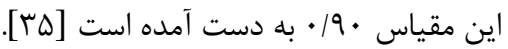

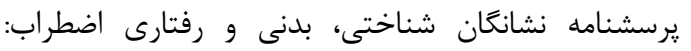

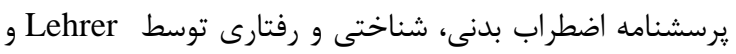
Woolfolk

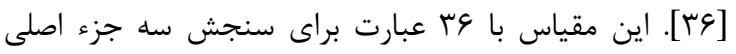



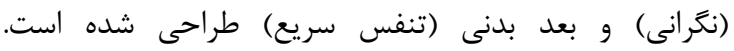

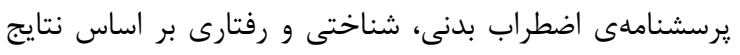

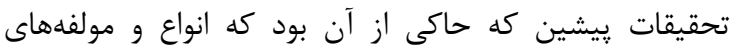

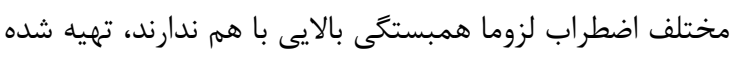

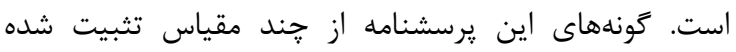

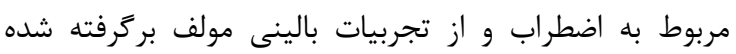

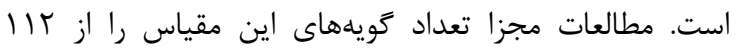

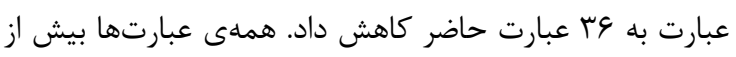

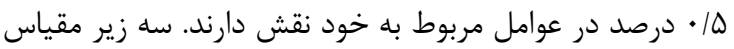

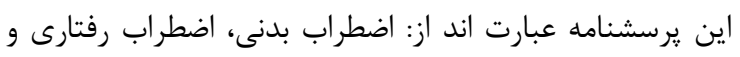

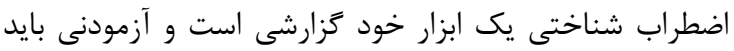

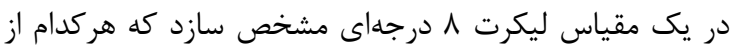

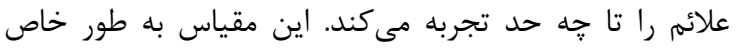

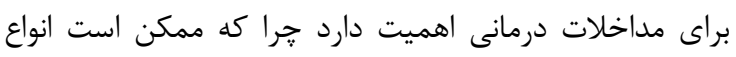

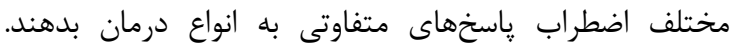

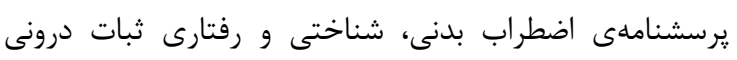

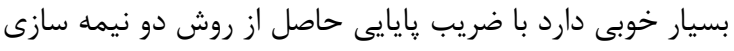

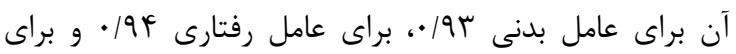

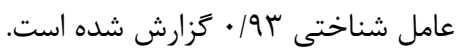

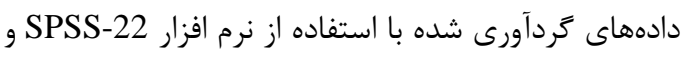

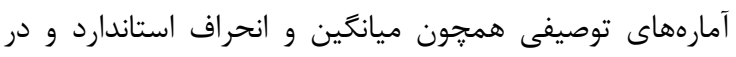

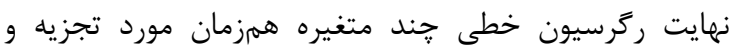

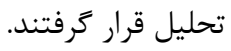

دادهها به طرحهاى توصيفى از نوع همبستكى تعلق دارد. به

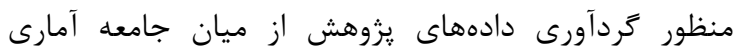
دانشجويان دانشكدههاى مختلف دانشكاه بوعلى سينا در ادر سال

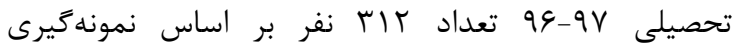

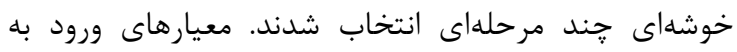

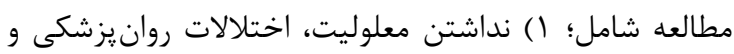
نورولوزيكى و آسيبهاى مغزى -جمجمهایى (Y) نداشتن اعتياد

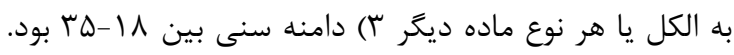

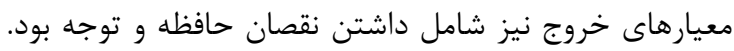

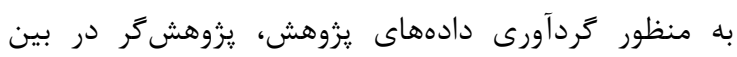

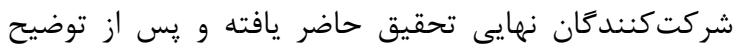

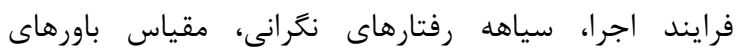

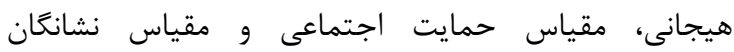



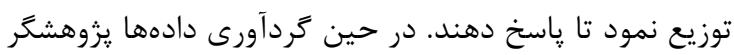

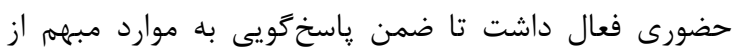

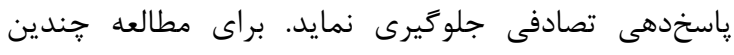
يرسشنامه استفاده شد.

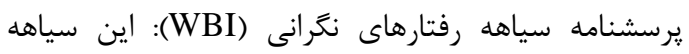

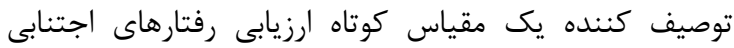

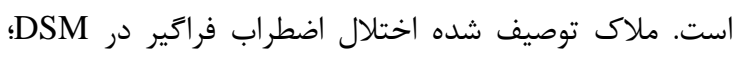
مدلهاى شناختى معاصر و ادبيات كسترده يِيرامون رفتارهاى نكَرانى مرتبط با GAD در شكل دهى مقيى مقياس مورد استفاده قرار

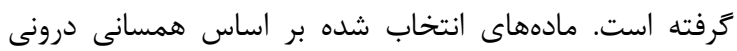



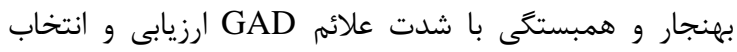

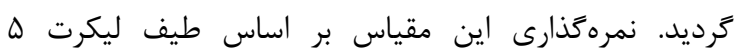

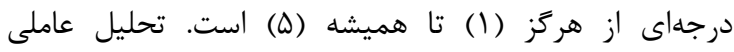

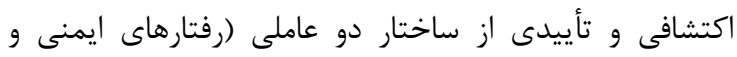

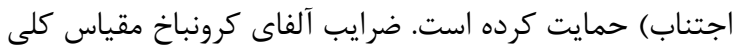

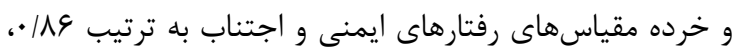

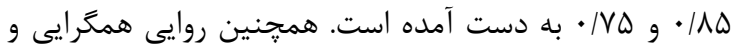

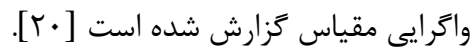



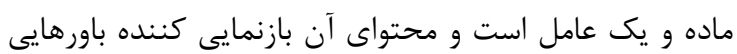

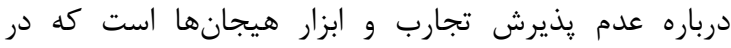





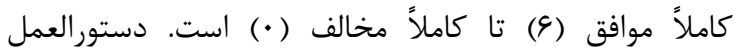

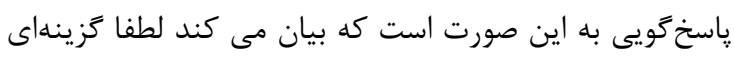

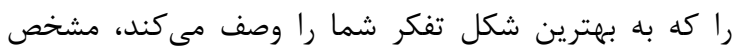

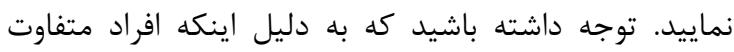

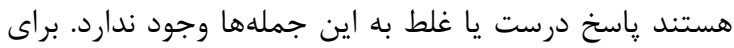

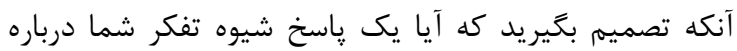


بافتهن.

نتايج جدول شماره r نشان دهنده وجود روابط معنادار بين

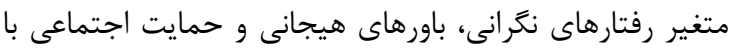

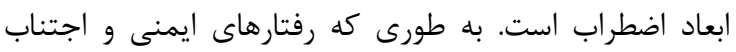

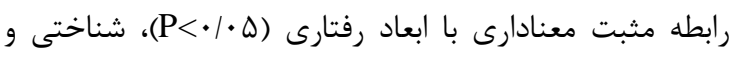



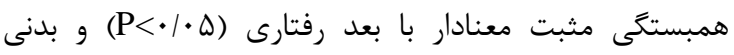
نشان داد. همبستكى منفى معنادارى بين حمايت

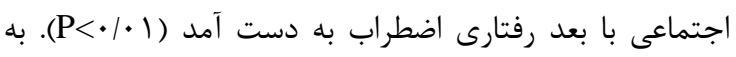

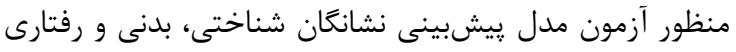



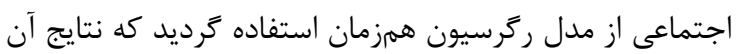
در جدول ب درج گرديده است.
شركتكنندكان يزوهش حاضر rاس نفر از دانشجويان رشتههاى مختلف از شاخههاى ادبيات و علوم انسانى، فنى

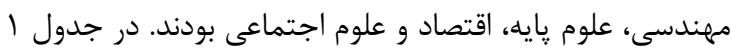

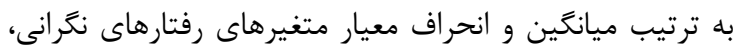
باورهاى هيجانى، حمايت اجتماعى و مقياس نشانگان شناختى، رئى بدنى و رفتارى اضطراب درج گرديده است. به منظور آزمون فرضيههاى يزوهش مبنى اصنى بر دردئ اينكه بين

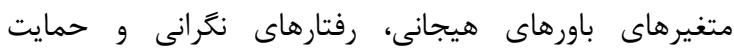

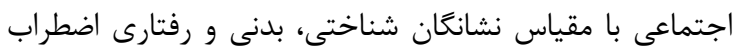

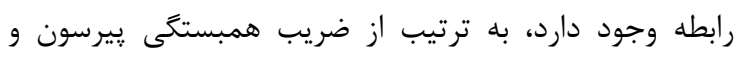

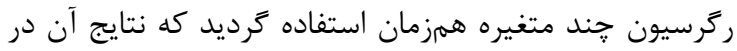

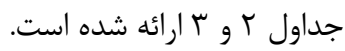

جدول ا: ميانخين و انحراف معيار متغيرها

\begin{tabular}{|c|c|c|c|}
\hline انحراف معيار 土 ميانگين & 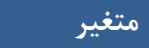 & انحراف معيار 土 ميانگين & متغير \\
\hline$r T / V \pm V / G$ & بعد رفتارى & $r r / \Delta \pm g / 1$ & رفتارهاى ايمنى \\
\hline$T r / \cdot \pm 1 \cdot / T$ & بعد شناختى & $9 / \Delta \pm r / r$ & اجتناب \\
\hline$r q / f \pm \mid r / l$ & بعد بدنى & $f \cdot / V \pm q / V$ & باورهاى هيجانى \\
\hline$r / / T \pm F / T$ & سن & $r / / l \pm \Delta / q$ & حمايت اجتماعى \\
\hline
\end{tabular}

\begin{tabular}{|c|c|c|c|c|c|c|c|}
\hline v & 9 & $\Delta$ & f & $r$ & r & 1 & متغير ها \\
\hline & & & & & & 1 & رفتارهاى ايمنى \\
\hline & & & & & 1 & • & اجتناب \\
\hline & & & & 1 & $\cdot / r^{* * * * *}$ & . $/ r$ & باورهاى هيجانى \\
\hline & & & 1 &.$/ 1 \pi$ & $-\cdot / \cdot r$ & $-\cdot 111$ & حمايت اجتماعى \\
\hline & & 1 & $-\cdot / 1 \Lambda^{* * *}$ & $\cdot 119^{*}$ & $\cdot / \Gamma \Lambda^{* * *}$ & $\cdot / 19^{*}$ & بعد رفتارى \\
\hline & 1 & $\cdot / \Delta \Delta^{* * *}$ & $.1 \cdot 1$ &.$/ 1 r$ & $\cdot / \Gamma \Delta^{* * * *}$ & $\cdot / \pi 9^{* * *}$ & بعد شناختى \\
\hline 1 & $\cdot|9|^{* * *}$ & $\cdot|9|^{4 * *}$ & $\cdot / \cdot r$ & $\cdot|r|^{* * * *}$ & $\cdot / r r^{\text {***** }}$ & $\cdot /\left.r\right|^{* * * *}$ & بعد بدنى \\
\hline
\end{tabular}

\begin{tabular}{|c|c|c|c|c|c|c|c|c|c|c|}
\hline $\mathbf{F}$ & $\mathbf{R}^{2}$ & $\mathbf{R}$ & $\mathbf{P}$ & $\mathbf{T}$ & Beta & SE & B & متغير بيشبين & متغير ملاك & مدل \\
\hline $11 / \Delta F$ & .119 & . & $\begin{array}{l}. / 9 F F \\
.1 \% \\
.1 \cdot 90 \\
.1 \cdot .9\end{array}$ & $\begin{array}{l}. / 49 \\
\text { F/9R } \\
1 / 9 V \\
-r / V V\end{array}$ & $\begin{array}{l}\cdot / \cdot r \\
\cdot / r r \\
\cdot / 11 \\
-\cdot / 18\end{array}$ & $\begin{array}{l}\cdot / \cdot 1 \\
\cdot / 4 T \\
\cdot 1 \cdot 0 \\
\cdot 1 \cdot 1\end{array}$ & $\begin{array}{l}\cdot 1 \cdot 4 \\
1 / \cdot 9 \\
\cdot 1 \cdot 1 \\
-\cdot / 4 r\end{array}$ & باورهاي & بعد رفتارى & 1 \\
\hline $9 / 11$ & .119 & $\cdot / 4$ & $\begin{array}{l}\cdot / \cdot \cdot r \\
\cdot / \cdots \\
\cdot|r T| \\
\cdot \mid \Delta F F\end{array}$ & $\begin{array}{l}r / \cdot r \\
r / \Lambda \cdot \\
\cdot / 99 \\
\cdot 19 .\end{array}$ & $\begin{array}{l}\cdot / 4 \cdot \\
\cdot / 49 \\
.1 \cdot 9 \\
\cdot / \cdot 4\end{array}$ & $\begin{array}{l}\cdot / 11 \\
\cdot / r \cdot \\
\cdot / \cdot V \\
\cdot / 11\end{array}$ & $\begin{array}{l}\cdot / T F \\
1 / 10 \\
.1 \cdot V \\
.1 \cdot 9\end{array}$ & باورايت اجتار هاى ايمنى اجتاب & بعد شناختى & r \\
\hline$\Lambda / T \Delta$ &.$/ 1 f$ & $\cdot / r V$ & $\begin{array}{l}\cdot 1 \cdot 4 T \\
\cdot 1 \cdot .1 \\
\cdot 1 \cdot 40 \\
.1099\end{array}$ & $\begin{array}{l}1 / 99 \\
r / 0 . \\
r / 1 T \\
. / 0 T\end{array}$ & $\begin{array}{l}\cdot / 1 Y \\
\cdot / 4 F \\
\cdot / 1 F \\
.1 \cdot r\end{array}$ & $\begin{array}{l}.114 \\
.149 \\
.1 .9 \\
.114\end{array}$ & $\begin{array}{l}\cdot 1 / 99 \\
1 / 48 \\
\cdot 119 \\
.1 \cdot 8\end{array}$ & باورايت اجتار هاب ايمنى & بعد بدنى & r \\
\hline
\end{tabular}


رفتارى و بدنى اضطراب رابطه مثبت دارند. يافتهها همسو با در مدلهاى شناختى و رفتارى است كه بيان شده است كه

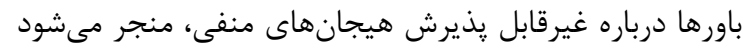

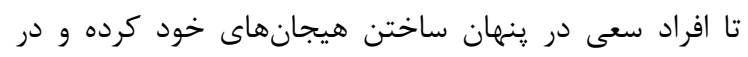

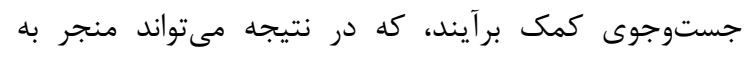

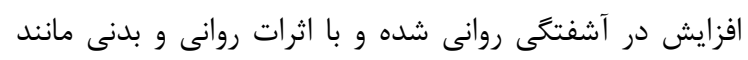
خستكى، مشكلات تمركز/ حافظه، اختلال روده و غيره مى

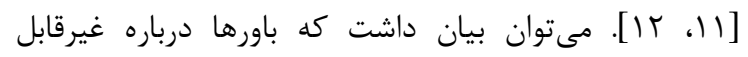
يذيرش بودن هيجانهاى منفى، شكلى از كمال

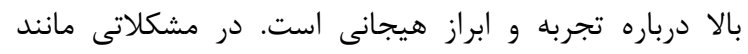

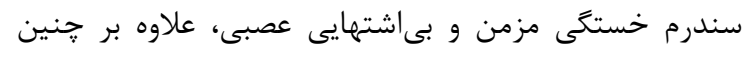



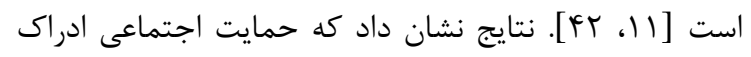

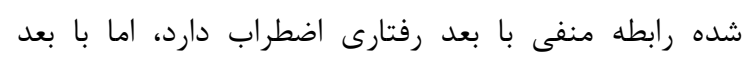
شناختى و بدنى رابطهاى نشان نداد. به طور مشابه، مطالعه فرا

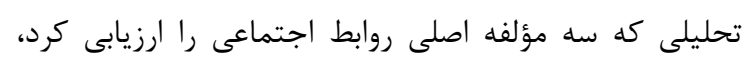

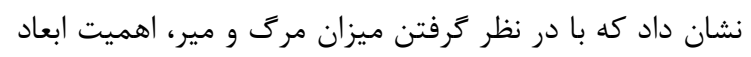

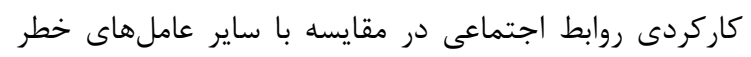

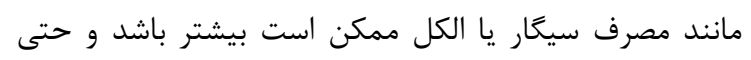

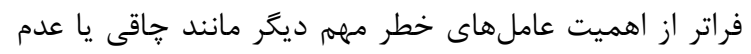

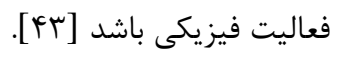

\section{نتيجه كيرى}

در مجموع يافتههاى يزوهش حاضر نشان داد كه باورهاى

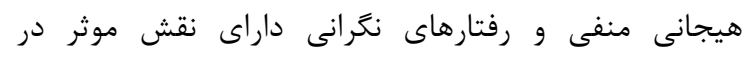

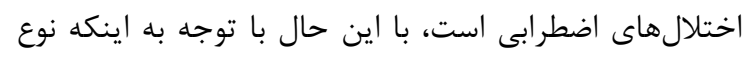

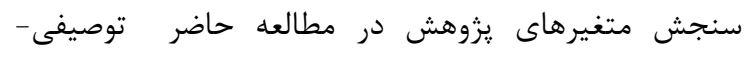

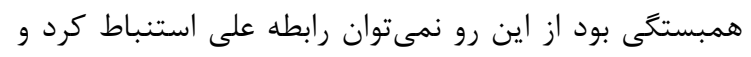

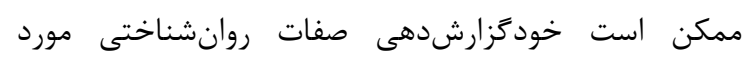

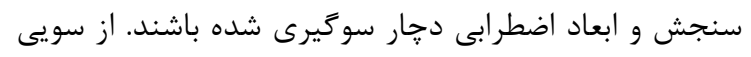

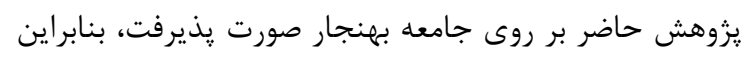

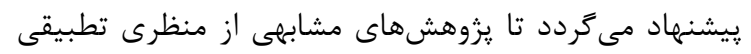

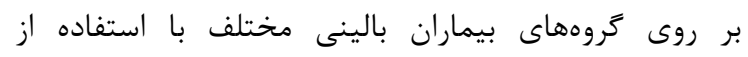

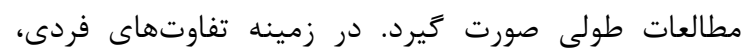



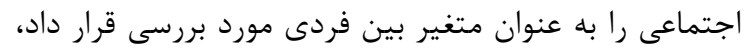

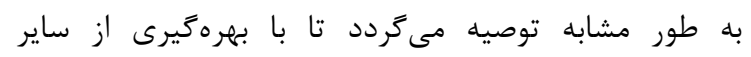

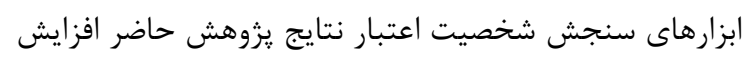



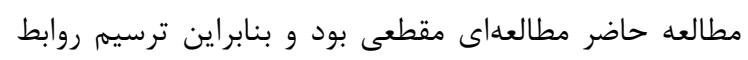

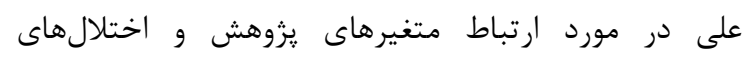

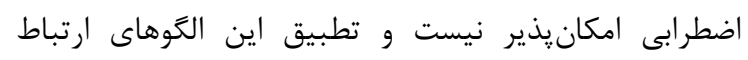
نيازمند مطالعات بيشترى است تا اعتبار بيشترى براى ئرئ يافتههاى يزوهش حاضر به دست آيد.
نتايج معادله ركرسيون در جدول r در مدل | نشان

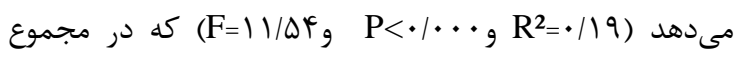
متغيرهاى ييشبين اجتناب و حمايت اجتماعى با مجذور

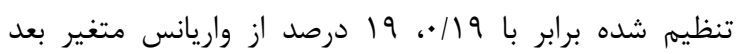

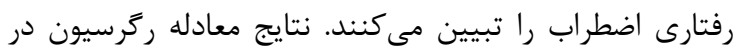

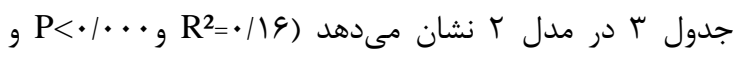

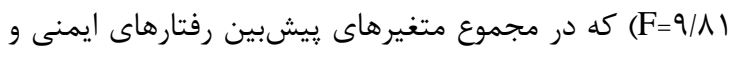

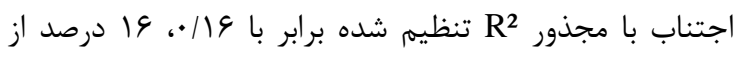

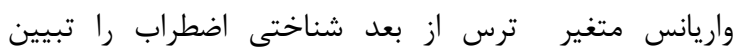



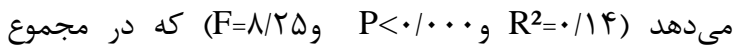



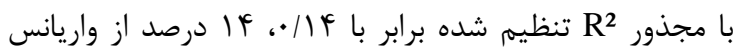
متغير بعد بدنى اضطراب را تبيين مى كنند.

بكث اضطراب پاسخى بهنجار نسبت به تهديد يا استرس است،

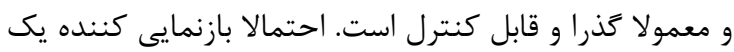

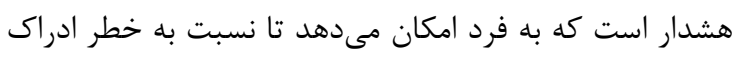
شده واكنش فيزيكى نشان دهد [rV]

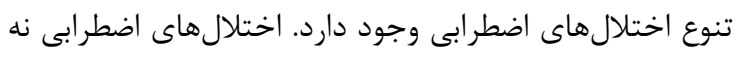



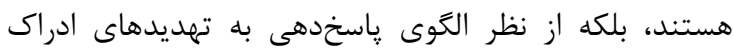

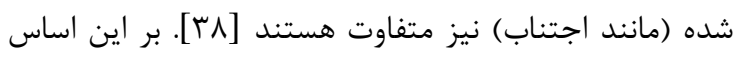

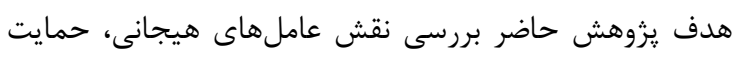

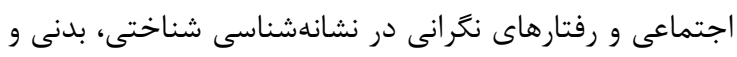

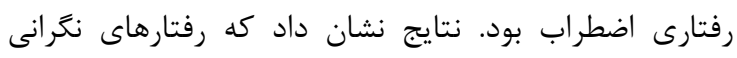

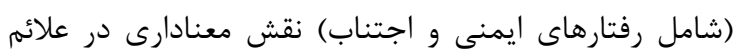

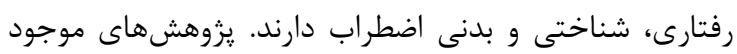

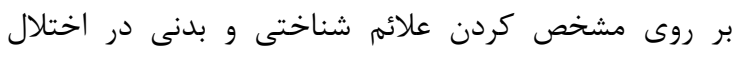

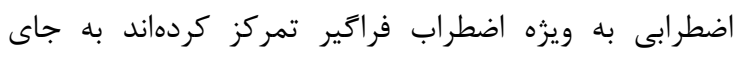

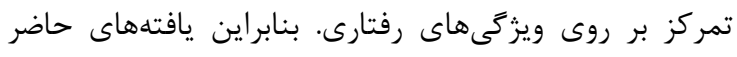

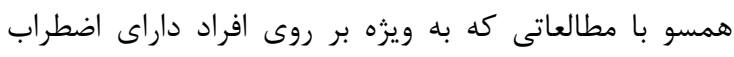

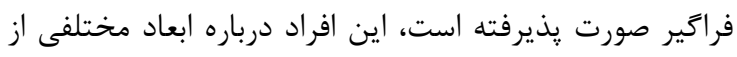

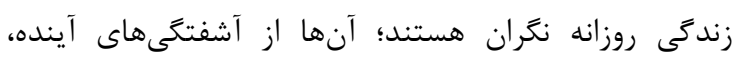

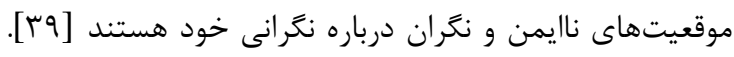
در نتيجه اين موضوع ممكن است منجر به اشكال ثابت و و

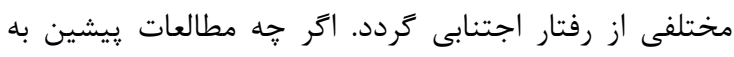

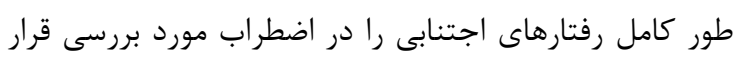

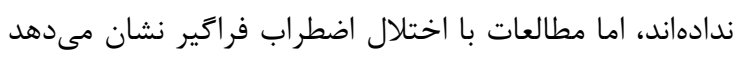

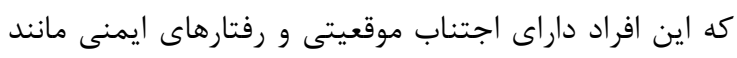
وارسى وسواسى، اطمينان يابى مجدد، برنامهريزى و و ساير إنى

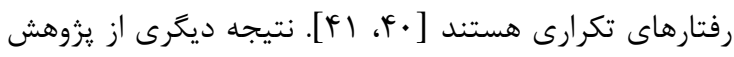
حاضر نشان داد كه باورهاى هيجانى منفى با علائم شناختى، 

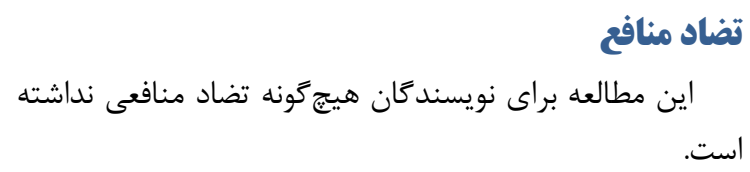

$$
\begin{aligned}
& \text { تشكر و قلروفاذى } \\
& \text { بدين وسيله از كليه دانشجويان دانشعاه بوعلى سينا كه در } \\
& \text { انجام يزوهش، نويسندكان را يارى نمودند تشكر و قدردانى به } \\
& \text { عمل مى آيد. }
\end{aligned}
$$

\section{REFERENCES}

1. American Psychiatric Association. Diagnosticand Statistical Manual of Mental Disorders DSM-5. 5th ed. Washington, D.C.: American Psychiatric Publishing 2013.

2. Horney K. The neurotic personality of our time 1937.

3. Hamilton M. The assessment of anxiety states by rating. $\mathrm{Br}$ J Med Psychol.1959; 32:50-5.

4. Barrett ES. Anxiety and impulsiveness: toward a neuropsychological model. In: \& Spielberger CD, editor. Anxiety: Current trends in theory and research. New York: Academic Press 1972.

5. Schwartz GE, Davidson RJ, Goleman DJ. Patterning of cognitive and somatic processes in the self-regulation of anxiety: effects of mediation versus exercise. Psychosom Med. 1978; 40:321-8.

6. Jarvis M. Sport psychology. New York: Routledge 2002.

7. Parnabas VA, Mahmood Y, Ampofo-Boateng K. Cognitive, somatic and negative coping strategies among Malaysian athletes. European Journal of Social Sciences. 2009; 9:387-98.

8. Weems CF, Silverman, WK. Anxiety disorders. In T. P. Beauchaine \& S. P. Hinshaw (Eds.), Child and adolescent psychopathology (pp. 447-476). Hoboken, N.J.: Wiley 2008.

9. Small GW. Recognizing and treating anxiety in the elderly. J Clin Psychiatry. 1997; 58: 41-47.

10. Suveg C, Sood E, Comer JS, Kendall PC. Changes in emotion regulation following cognitive-behavioral therapy for anxious youth. J Clin Child Adolesc Psychol. 2008; 38, 390-401.

11. Surawy C, Hackmann A, Hawton K, Sharpe M. Chronic fatigue syndrome: a cognitive approach. Behav Res Ther. 1995; 33:535-44.

12. Ali A, Toner BB, Stuckless N, Gallop R, Diamant NE, Gould MI, Vidins EI. Emotional abuse, self-blame, and selfsilencing in women with irritable bowel syndrome. Psychosom Med. 2000; 62:76-82.

13. Clark DM, Wells A. A cognitive model of social phobia. In: Heimberg RG, Liebowitz MR, Hope DA, Schneier FR, editors. Social phobia: diagnosis, assessment and treatment. New York: Guilford Press 1995.

14. Cramer KM, Gallant MD, Langlois MW. Self-silencing and depression in women and men: Comparative structural equation models. Pers Individ Dif. 2005; 39:581-92.

15. Ehlers A, Clark DM. A cognitive model of posttraumatic stress disorder. Behav Res Ther. 2000; 38: 319-45.

16. Linehan M. Cognitive-behavioural treatment of borderline personality disorders. New York: Guilford Press 1993.

17. Corstorphine E. Cognitive-emotional-behavioural therapy for the eating disorders: working with beliefs about emotions. Eur Eat Disord Rev. 2006 14: 448-61.

18. Kennedy-Moore E, Watson JC. How and when does emotional expression help? Rev Gen Psychol. 2001; 5:187-212.

19. Wenzlaff RM, Wegner DM. Thought suppression. Annu Rev Psychol. 2000; 51:59-91.

20. Mahoney AEJ, Hobbs MJ, Newby JM, Williams AD, Sunderland M, Andrews G. The Worry Behaviors Inventory: Assessing the behavioral avoidance associated with generalized anxiety disorder. J Affect Disord. 2016; 203, 256-264.

21. Schut AJ, Castonguay LG, Borkovec TD, Compulsive checking behaviors in generalized anxiety disorder. J Clin Psychol. 2001; 57: 705-715.

22. Cuming S, Rapee RM, Kemp N, Abbott MJ, Peters L, Gaston JE. A selfreport measure of subtle avoidance and safety behaviors relevant to social anxiety: development and psychometric properties. J Anxiety Disord. 2009; 23, 879883
23. Ottenbreit ND, Dobson KS. Avoidance and depression: the construction of the cognitive-behavioral avoidance scale. Behav Res Ther. 2004; 42, 293-313.

24. Sexton KA, Dugas MJ. The Cognitive Avoidance Questionnaire: validation of the English translation. J Anxiety Disord. 2008; 22, 355-370. DOI:10.1016/j.janxdis. 2007.04.005

25. Lakey B, Orehek E. Relational regulation theory: a new approach to explain the link between perceived social support and mental health. Psychol Rev 2011; 118: 482.

26. Barth J, Schneider S, Von K€anel R. Lack of social support in the etiology and the prognosis of coronary heart disease: a systematic review and meta-analysis. Psychosom Med 2010; 72: 229-38. DOI:10.1097/PSY.0b013e3181d01611

27. DiMatteo MR. Social support and patient adherence to medical treatment: a meta-analysis. Health Psychol. 2004; 23: 207.

28. Lett HS, Blumenthal JA, Babyak MA, Catellier DJ, Carney RM, Berkman LF, et al. Social support and prognosis in patients at increased psychosocial risk recovering from myocardial infarction. Health Psychol. 2007; 26:418. DOI:10.1037/0278-6133.26.4.418

29. Kwong EW, Kwan AY-H. Stress-management methods of the community-dwelling elderly in Hong Kong: Implications for tailoring a stress-reduction program. Geriatric Nursing 2004; 25: 102-106.

30. Alipour F, Sajadi H, Forouzan AS, Biglarian A. The role of social support on quality of life of elderly. Social Welfare Quarterly 2009; 9:147-65. (Persian)

31. Brummett BH, Mark DB, Siegler IC, Williams RB, Babyak MA, Clapp-Channing NE, et al. Perceived social support as a predictor of mortality in coronary patients: effects of smoking, sedentary behavior, and depressive symptoms. Psychosom Med 2005; 67(1):40-5.

32. Martens R, Vealey RS, Burton D. Competitive anxiety in sport. Champaign, Illinois: Human Kinetics 1990.

33. Jin X, Cao X, Zhong M, Chen L, Zhu X, Yao S, Yi J. The psychometric properties of the Cognitive-Somatic Anxiety Questionnaire in Chinese undergraduate students and clinical patients. Comprehensive Psychiatry 2014; 55: 1751-1756.

34. Rimes KA, Chalder T. The Beliefs about Emotions Scale: Validity, reliability and sensitivity to change. J Psychosom Res. 2010; 68: 285-292. DOI: 10.1016/j.jpsychores.2009. 09.014

35. Kliem S, Moßle T, Rehbein F, Hellmann DF, Zenger M, Brahler E. A brief form of the Perceived Social Support Questionnaire (F-SozU) was developed, validated, and standardized. J Clin Epidemiol 2014; 68(5):551-62. DOI:10.1016/j.jclinepi.2014.11.003

36. Lehrer PM, Woolfolk RL. Self-report assessment of anxiety: Somatic, cognitive and behavioral modalities. Behavioral Assessment 1982; 4: 167-111.

37. Gordon RP, Brandish EK, Baldwin DS. Anxiety disorders, post-traumatic stress disorder, and obsessive compulsive disorder. Psychiatric Disorders (in press).

38. Berle D, Starcevic V, Milicevic D, Hannan A, Dale E, Skepper B, Viswasam K, Brakoulias V. The structure and intensity of self-reported autonomic arousal symptoms across anxiety disorders and obsessive-compulsive disorder. J Affect Disord 2016; 199: 81-86. DOI:10.1016/j.jad.2016.04.010

39. Wells A, Carter K., Further tests of a cognitive model of generalized anxiety disorder: metacognitions and worry in GAD, panic disorder, social phobia, depression, and nonpatients. Behav Ther 2001; 32: 85-102.

40. Beesdo-Baum K, Jenjahn E, Hofler M, Lueken U, Becker ES, Hoyer J. Avoidance, safety behavior, and reassuranceseeking in generalized anxiety disorder. Depress Anxiety. 
2012; 29: 948-957. DOI:10.1002/da.21955

41. Coleman SL, Pieterfesa AS, Holaway RM, Coles ME, Heimberg RG. Content and correlates of checking related to symptoms of obsessive compulsive disorder and generalized anxiety disorder. J Anxiety Disord 2011; 25: 293-301. DOI: 10.1016/j.janxdis. 2010.09.014
42. Schmidt U, Treasure J. Anorexia nervosa: valued and visible. A cognitive-interpersonal maintenance model and its implication for research and practice. Br J Clin Psychol 2006; 45: 343-66.

43. Ferster CB. A functional analysis of depression. Am Psychol 1973; 28: 857-70. 